\title{
Thermally Limited Force Microscopy on Optically Trapped Single Metallic Nanoparticles
}

\author{
Gabriel Schnoering, ${ }^{1}$ Yoseline Rosales-Cabara, ${ }^{1}$ Hugo Wendehenne, ${ }^{1, \dagger}$ \\ Antoine Canaguier-Durand, ${ }^{2}$ and Cyriaque Genet ${ }^{1}{ }^{*}$ \\ ${ }^{1}$ ISIS and icFRC, University of Strasbourg and CNRS, 8 allée Gaspard Monge, 67000 Strasbourg, France \\ ${ }^{2}$ Laboratoire Kastler-Brossel, UPMC-Sorbonne Universités, CNRS, ENS-PSL Research University, \\ Collège de France, 4 place Jussieu, 75005 Paris, France
}

(Received 23 January 2019; published 8 March 2019)

\begin{abstract}
We propose and evaluate a new type of optical force microscope based on a standing-wave optical trap. Our microscope, calibrated in situ and operating in a dynamic mode, is able to trap, without heating, a single metallic nanoparticle of $150 \mathrm{~nm}$ that acts as a highly sensitive probe for external radiation pressure. An Allan-deviation-based stability analysis of the setup yields an optimal $0.1-\mathrm{Hz}$ measurement bandwidth over which the microscope is thermally limited. Over this bandwidth, and with a genuine sine-wave external drive, we demonstrate an optical force resolution down to $3 \mathrm{fN}$ in water at room temperature with a dynamical range for force detection that covers almost 2 orders of magnitude. This resolution is reached in both the confined regime and the freely diffusing regime of the optical trap. In the latter, we measure induced displacements of $10^{-11} \mathrm{~m}$ on the trapped nanoparticle spatially confined within less than $25 \mathrm{~nm}$ along the optical axis.
\end{abstract}

DOI: 10.1103/PhysRevApplied.11.034023

\section{INTRODUCTION}

Optical traps have become central experimental tools for measuring forces at the nanoscale with outstanding positional and force resolutions. Because of their small sizes, optically trapped objects have enabled thermally limited sensitivity, in particular in liquids [1-4]. These remarkable features have been exploited in a vast variety of contexts, ranging from biology [5] and nonequilibrium physics [6] to optomechanics [7]. Recent work has demonstrated how nonconservative optical force fields can be measured and spatially resolved with use of optically trapped dielectric particles, leading to the study of nontrivial Brownian-type motions [8-10]. In this context, use of metallic nanoparticles (NPs) would offer new opportunities considering all the specific modes of actuation and control that could be implemented on metallic nano-objects through their high extinction cross sections [11-16]. However, it remains difficult to achieve three-dimensional stable optical trapping of large metallic NPs (i.e., radii larger than $50 \mathrm{~nm}$ ) in fluids in standard-laser-intensity conditions [17-19]. This difficulty remains a strong limitation despite the potential offered by metallic NPs, in particular in the context of biophysics [20], nanosensing [21], and spectroscopy [22].

\footnotetext{
*genet@unistra.fr

†Present address: Universität Konstanz, 78464 Constance, Germany
}

In this paper, we propose a setup where stable trapping conditions for single large Au NPs can be reached at low laser power. In addition, our setup offers, by construction, the possibility to inject an additional laser beam that can exert, in a perfectly controllable way, radiation pressure on the trapped NP along the optical axis and independently of the restoring force at play inside the trap. Importantly, all this is achieved with negligible heating, as discussed below.

To qualify our setup as a force microscope, important features are implemented. The additional pushing laser beam is injected inside the trap in such a way as to generate an optical force field as uniform as possible. Having the strength of the external force exerted on the NP constant throughout the diffusion volume of the NP inside the trap is a central feature of our scheme that stands out from force measurements performed using total-internalreflection microscopes. It allows us to operate our setup in a dynamical mode where the pushing laser, harmonically driven at a fixed frequency, leads to a modulated force signal independent of the instantaneous position of the NP inside the trap. In such conditions, the dynamical mode operation is particularly appealing from a calibration point of view since it does not require any static force calibration procedure [23-27]. In our experiments, the external force is directly measured from the power spectral density (PSD) of the motion of the trapped NP at the modulation frequency. Operating with a uniform force field, one 
simply requires a positional calibration of the optical trap, obtained from the fluctuation-dissipation theorem (FDT) [28]. This straightforward approach is clearly an advantage of our method, considering that a static force calibration is, in general, challenging to perform within the limits of stability of the setup.

Discussing such limits is important since they play a crucial role in the determination of the force resolution level [29-32]. They yield the optimal (maximal) time $\tau_{\text {opt }}$ over which a measurement remains thermally limited. In our work, these limits are properly identified through a global stability analysis of the setup. The acquisition of the positional PSD of the trapped NP over $\tau_{\text {opt }}$ thus directly leads to the determination of the smallest external force $F_{\text {opt }}$ measurable with our setup. Shorter than the duration over which the NP can remained trapped, $\tau_{\text {opt }}$ warns against the appealing but misleading possibility to increase resolution by reducing the spectral noise density through an average of independent sequences of measurements extracted from acquisition time series longer that $\tau_{\text {opt }}$. At the level of a single measurement performed over $\tau_{\text {opt }}$, the signal associated with the external drive is superimposed on contributions not only from thermal fluctuations but also from other (uncharacterized) noise sources. As detailed below, the stability analysis sets for our experiments a resolution criterion at twice the thermal limit that leads us to demonstrate an external-optical-force resolution of $3 \mathrm{fN}$ in water at room temperature within a measurement time $\tau_{\text {opt }}=10 \mathrm{~s}$.

\section{STANDING-WAVE OPTICAL TRAP}

Our optical trap configuration, schematized in Fig. 1, consists in the focusing of a trapping laser beam of $\lambda_{T}=$ $785 \mathrm{~nm}$ into a $120-\mu \mathrm{m}$ fluidic cell, entering with a mean intensity of $24.5 \mathrm{~mW}$ through a water-immersion objective on top of an end mirror. The beam largely overfills the objective pupil and its power is estimated to be approximately $10 \mathrm{~mW}$ at the waist. This mirror is highly reflecting at $\lambda_{T}$ and therefore induces a standing-wave pattern inside the cell. In such a counterpropagating-beam configuration, the incident and reflected scattering forces acting on the NP practically compensate each other, and can therefore be easily overcome by the gradient force induced by the focusing effect of the objective [33]. This configuration yields a balance of forces appropriate for trapping metallic NPs of radius larger than $50 \mathrm{~nm}$, an interesting asset when one is aiming at measuring radiation-pressure forces. Such capacity is not found for conventional single-beam traps, where the scattering forces tend to push away from the waist such large metallic NPs. We check experimentally that replacing the end mirror by a glass slide does not allow trapping of the $\mathrm{Au}$ NPs. This is in agreement with calculations that cannot find any stable position for such an $\mathrm{Au}$ NP within a propagating Gaussian beam determined from

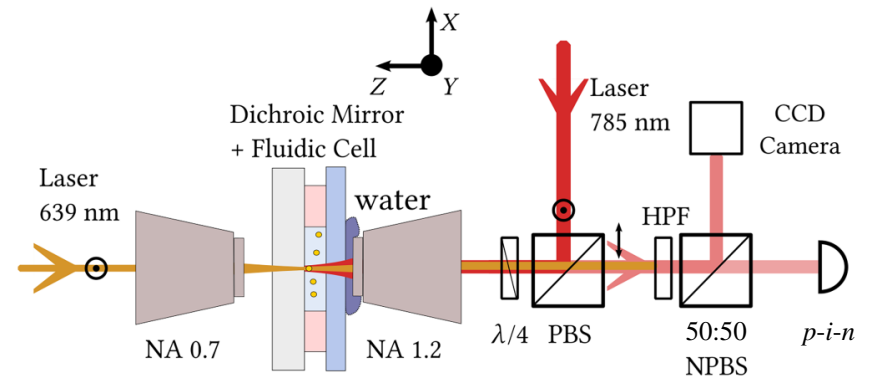

FIG. 1. The experimental setup. Gold nanospheres (BBI Solutions) are trapped by a linearly polarized near-IR laser (785-nm, 45-mW Spectra-Physics Excelsior laser diode, optically isolated) sent to a water-immersion objective (NA 1.2, $\times 100)$ through a polarizing beam splitter (PBS) and a half-wave plate $(\lambda / 4)$ with a power of $24.5 \mathrm{~mW}$ at the entrance of the objective. A standingwave optical trap is formed by the reflection of the laser beam on a dichroic mirror (cutoff at $700 \mathrm{~nm}$ ). The reflected intensity $I(t)$ varies linearly with the nanosphere displacement $z(t)$ inside the trap and is collected and recorded by a $p-i-n$ photodiode (Thorlabs det10A), while a CCD camera is used in the other port of the nonpolarizing beam splitter (NPBS) for imaging. A second beam (639-nm, 70-mW Thorlabs laser diode, linearly polarized) of low power $(400 \mu \mathrm{W})$ is injected inside the trap collinearly with the trapping beam but from behind the dichroic mirror with use of a dry objective (NA $0.7, \times 60$ ). This second beam is not expanded and does not fill the entrance pupil of the objective. It is hence only weakly focused in front of the trapped bead and can be used to push the bead along the optical axis, minimizing any gradientforce effect. To avoid any intensity signature of the modulated signal on the $p-i-n$ photodiode, a $650-\mathrm{nm}$ high-pass filter (HPF) is added.

our experimental conditions for wavelength and numerical aperture (NA) [34].

We monitor the instantaneous position $z(t)$ of the NP by recording the trapping laser light scattered by the NP in the forward direction and reflected back toward the $p-i-n$ detector; see Fig. 1 . In the Fourier domain, the motional dynamics of the NP $\hat{z}(f)$ is described by its PSD $S_{z}(f)=2|\hat{z}(f)|^{2}$ [35]. In the low-Reynolds-number conditions of our experiments, the overdamped displacement of the NP along the optical axis of the trap obeys the spectral Langevin equation $\hat{z}(f)=\chi(f) \hat{F}_{\text {th }}(f)$, where $\chi(f)$ is the mechanical susceptibility of the NP inside the trap and $\hat{F}_{\text {th }}(f)$ is the Langevin force responsible for the Brownian motion of the NP. Assuming that the response of the trapped bead is harmonic, the susceptibility

$$
\chi(f)=\frac{1}{\kappa_{T}-i 2 \pi \gamma f}
$$

is Lorentzian, where $\gamma=6 \pi \eta R$ is the Stokes drag and $\eta \sim$ $10^{-3} \mathrm{~Pa} / \mathrm{s}$ is the dynamical viscosity of water at room temperature. The stiffness of the harmonic trap $\kappa_{T}=2 \pi \gamma f_{T}$ is characterized by a roll-off frequency $f_{T}$. 
At thermal equilibrium, the (one-sided) Langevin force spectral density is given by the FDT with $S_{\mathrm{th}}(f)=4 k_{B} T \gamma$. The bead dynamics is entirely driven by thermal fluctuations that have a broad Gaussian-white-noise spectrum. The PSD

$$
S_{z}(f)=\frac{D}{\pi^{2}\left(f^{2}+f_{T}^{2}\right)}
$$

thus spectrally describes the motional response of the trapped NP under the action of thermal forces with diffusion coefficient $D=k_{B} T / \gamma$.

The PSD $S_{z}(f)$ is determined after calibration of the intensity PSD $S_{I}(f)$ against the known properties of the fluid and under the assumption that the recorded intensity $I(t)$ is linear with the displacement $z(t)$ of the NP. This assumption holds in our experiment because the bead displacements in the trap are small and the measured PSD follows the Lorentzian fit that uses a linear restoring force.

The calibration factor is obtained by our fitting the experimental PSD by the Lorentzian model of Eq. (2). The fit uses only points above $10 \mathrm{~Hz}$, where it is reasonable to assume that all the displacement contributions (aside from the external-force peak) originate from thermal fluctuations. This provides best-fitted values for $D$ and $f_{T}$, and from the diffusion coefficient $D_{\mathrm{FDT}}=k_{B} T / \gamma$ calculated from the FDT (assuming known temperature and viscosity), the calibration factor is simply $\beta=D_{\mathrm{FDT}} / D$ and the calibrated PSD $S_{z}(f)=\beta^{2} S_{I}(f)$. For the one-sided PSD in Fig. 2, we extract a best-fitted roll-off frequency $f_{T}=8$ $\mathrm{kHz}$ and a calibration factor $\beta=1.2 \times 10^{-7} \mathrm{~m} / \mathrm{V}$.

Because the trap stiffness $\kappa_{T}$, which is directly proportional to the field intensity, depends on the viscosity $\eta(T)$, it is clear that a wrong estimation of the fluid temperature can impact the calibration of the setup and the force measurements. Optical powers at the waists of tightly focused light beams can reach levels on the order of megawatts per square centimeter, and metallic objects are subjected to strong elevations of temperature, around $1500 \mathrm{~K} / \mathrm{W}$ for $\mathrm{Au}$ NPs of $150 \mathrm{~nm}$ at $\lambda_{T}$ (see Supplemental Material Sec. C). This would correspond for our experiments to an increase in temperature of approximately $40 \mathrm{~K}$, which gives, for water, a change in viscosity by a factor of 2 . To check, and if necessary, estimate such unwanted heat contributions, we vary the trapping-beam intensity for different $\mathrm{Au}$ NPs stably trapped, and at different mirror-waist distances. As discussed in detail in Supplemental Material Sec. C, no deviations from linearity for trap stiffness as a function of the laser power are observed, suggesting a constant surrounding viscosity for all trapping-laser intensities. Importantly, therefore, heating of the trapped $\mathrm{Au}$ NP in our system, if present, has a negligible impact on calibration of the setup and measurement of an external force.

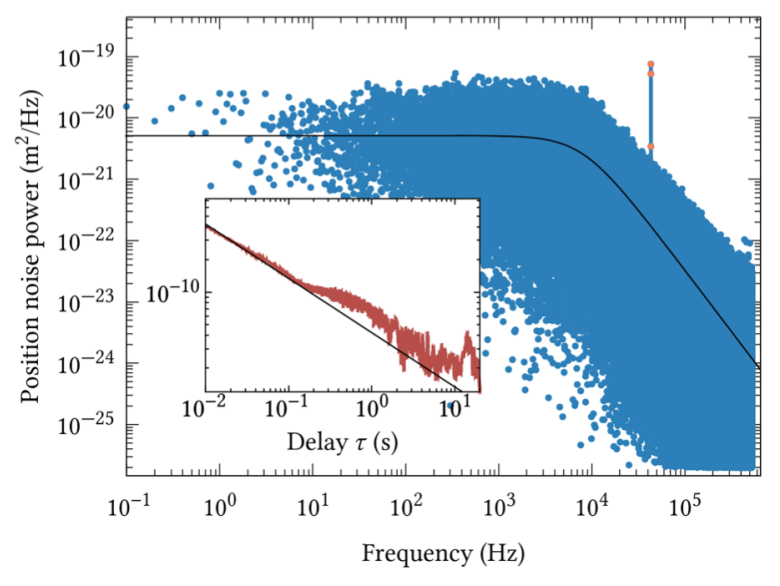

FIG. 2. One experimental (one-sided) PSD acquired over $\tau_{\text {opt }}=10 \mathrm{~s}$ at $1 \mathrm{MHz}$ is shown in the main panel, with roll-off frequency $f_{T}=8 \mathrm{kHz}$. The corresponding Lorentzian fit is shown in black. The external-drive amplitude, with a modulation ratio of 0.3 , has a strong spectral signature around $f_{0}=43333 \mathrm{~Hz}$. The height of the peak is large and well above the PSD variance. The spectral height of this contribution leads to a measure of the force via the trap calibration. The Allan deviation $\sigma_{z}(\tau)$ of a trapped gold nanosphere without external drive is shown in the inset. For time delays less than $0.1 \mathrm{~s}, \sigma_{z}(\tau)$ has a slope of $-1 / 2$. This, on a PSD plot, is white noise and corresponds to the plateau at low frequencies where the bead is trapped. For low frequencies (i.e., long time delays) a slight departure from the thermally driven dynamics of the bead appears. At $10 \mathrm{~s}$, it usually ranges between 1.2 and 2 depending on the trapped Au NP and its distance to the end mirror.

\section{TEST OF GLOBAL STABILITY: ALLAN-DEVIATION ANALYSIS}

As emphasized in Sec. I, the limited stability of the entire experimental setup in standard conditions puts an upper bound on the available measurement time. This is particularly true for our configuration, where the interferometric nature of our trap makes it very sensitive to all external perturbations, such as flow drift and evaporation inside the fluidic cell and vibrations. Although a single $\mathrm{Au}$ NP can be kept in the trap for minutes, the low frequency drift of our optofluidic system impacts the overall trapping dynamics. As a result of this uncontrolled drift, it is not possible to average a large number of measurements repeated for long acquisition times to increase the experimental sensitivity. This would misestimate the roll-off frequency.

In this context, it is important to determine the optimal data acquisition time $\tau_{\text {opt }}$ beyond which the various sources of noise have caused the entire setup to drift out of the thermodynamic response given by Eq. (2) $[29,36]$. To do so, we evaluate the Allan deviation $\sigma_{z}(\tau)$ for various NPs trapped for $1 \mathrm{~min}$ at different mirror-waist distances. A typical $\sigma_{z}(\tau)$ plot is shown in the inset in Fig. 2. The Allan deviation measures the standard deviation of the 
bead motion after it has been averaged for a time $\tau$. This analysis clearly reveals low-frequency-noise sources for acquisition times longer than $100 \mathrm{~ms}$. Nevertheless, their contribution remains limited up to $10 \mathrm{~s}$. For all the NPs measured, the differences between $\sigma_{z}(\tau)$ and the thermal limit at $10 \mathrm{~s}$ remained within ratios of 1.2 and 2 . This gives an optimal time $\tau_{\mathrm{opt}} \sim 10 \mathrm{~s}$ where noise sources remain limited throughout all measurements. The maximal deviation from the thermal limit, a factor of 2 after $10 \mathrm{~s}$, also sets a strong criterion for the minimal resolvable external force, as discussed later. All our measurements, as for the PSD displayed in Fig. 2, are performed over $\tau_{\text {opt }} \sim 10 \mathrm{~s}$, corresponding to an experimental bandwidth $\Delta f_{\mathrm{opt}}=0.1 \mathrm{~Hz}$.

Under such conditions of optimal bandwidth, the spatial confinement offered by the optical trap at thermal equilibrium is set by the equipartition result $\delta z=\sqrt{k_{B} T / \kappa_{T}}$. With the values extracted from the fit of the PSD in Fig. 2, we evaluate $\delta z=24 \mathrm{~nm}$. Considering that the trapping position is typically located approximately $2-3 \mu \mathrm{m}$ from the mirror surface, such a low $\delta z$ value leads to our ignoring any $z$-dependent surface-induced correction to $\eta$, which can safely be taken as its bulk value of approximately $10^{-3} \mathrm{~Pa} / \mathrm{s}$. Recalling that a fixed value for the viscosity is a necessary condition for the calibration procedure presented above, one understands that this $\delta z$ value is an important parameter to determine.

\section{THE FORCE MICROSCOPE}

Our force microscope, schematized in Fig. 1, consists in illumination of a Au NP optically trapped (in the standing wave of the trapping laser) with a second pushing laser that exerts an optical force on the NP. The wavelength $\lambda_{P}$ of the pushing laser is set from a Mie calculation that gives at $640 \mathrm{~nm}$ a maximum for the extinction coefficient of a $\mathrm{Au}$ $\mathrm{NP}$ of radius $R=75 \mathrm{~nm}$ illuminated by a plane wave. This allows for large radiation-pressure effects with low laser intensities (a mean intensity of $400 \mu \mathrm{W}$ at the entrance of the objective).

It is absolutely crucial that the pushing laser acts on the NP independently of the restoring force at play inside the trap. To this aim, we use a dichroic end mirror that reflects the trapping laser beam of $\lambda_{T}=785 \mathrm{~nm}$ while transmitting the pushing laser beam of $\lambda_{P}=639 \mathrm{~nm}$. The pushing laser beam comes from behind the mirror collinearly with respect to the optical axis of the trapping laser beam. It underfills a dry microscope objective (NA $0.7, \times 60)$ in such a way that, transmitted through the dichroic mirror, it is only slightly focused behind the trapped bead. Both gradient and scattering forces are at play, but we carefully check that the pushing laser beam never traps the NP and never perturbs the trap dynamics. We confirm numerically that at such a wavelength $\lambda_{P}$ and with such a NA, the pushing laser beam is not able to trap a Au NP of radius $R=75 \mathrm{~nm}$.
In the dynamical mode operation of the optical trap, the pushing-laser power is sinusoidally modulated at frequency $f_{0}$ around a mean value with $P_{P}=\langle P\rangle_{t}+$ $P_{\text {mod }} \cos 2 \pi f_{0} t$. A high-pass filter above $\lambda_{P}$ eliminates any contamination in the scattered $\lambda_{T}$ signal by the modulation of the pushing laser. The overdamped dynamics of the optically trapped NP is therefore simply determined by a static (dc) force component $F_{\mathrm{dc}}$ (proportional to $\langle P\rangle_{t}^{2}$ ) and a modulated (ac) force component $F_{\text {ac }}$ (proportional to $P_{\text {mod }}^{2}$ ), both added to the thermal Langevin force, as discussed in Supplemental Material Sec. D. This yields a spectral displacement

$$
\hat{z}(f)=\chi(f)\left\{\hat{F}_{\mathrm{th}}(f)+F_{\mathrm{dc}} \delta(0)+\frac{F_{\mathrm{ac}}}{2}\left[\delta\left(f-f_{0}\right)+\delta\left(f+f_{0}\right)\right]\right\}
$$

from which the contribution of the radiation pressure can be measured at the drive frequency. Experimentally, the output signal from the $p-i-n$ photodiode that records $z(t)$ is sent into a low-noise preamplifier. A high-pass filter with a cutoff frequency of $0.03 \mathrm{~Hz}$ removes from the signal the dc component of the force, which can then span the whole vertical resolution of the acquisition card with the bead dynamics that includes the ac force modulation. The measured signal is thereby not electronically limited.

The pushing laser beam is injected in such a way that the external force field induced by the pushing laser beam on the NP can be considered uniform throughout the volume of the optical trap. The forces exerted on the trapped NP are hence not modulated by its Brownian diffusion. This central feature gives Eq. (3) its simple structure with external-force components $\left(F_{\mathrm{dc}}, F_{\mathrm{ac}}\right)$ independent of $z(t)$. Position-dependent external forces, such as found with evanescent waves involved in total-internalreflection microscopy, would complicate the whole experiment. Equation (3) also implies that the pushing laser beam induces no additional heating effect. We carefully checked that this is the case, with no change observed in the trap stiffness with the external force on (see details in Supplemental Material Sec. C).

\section{FORCE MEASUREMENT, SENSITIVITY, AND RESOLUTION}

Under such sinusoidal modulation of the pushing laser, the spectral signature of the ac force component $F_{\mathrm{ac}}$ is a single resonant peak centered at the modulation frequency $f_{0}$ of the pushing laser. It is directly superimposed on the (one-sided) PSD $S_{z}^{d}(f)$ of the driven trapped bead as

$$
S_{z}^{d}(f)=\frac{1}{\left(f^{2}+f_{T}^{2}\right)}\left[\frac{D}{\pi^{2}}+\frac{F_{\mathrm{ac}}^{2}}{8 \pi^{2} \gamma^{2}} \delta\left(f-f_{0}\right)\right] .
$$

In a first series of experiments, we exert optical forces on the NP with a sufficiently strong $P_{\text {mod }}$ modulation so that the peak associated with $F_{\mathrm{ac}}$ clearly emerges above the 
PSD noise level. Because of the electronics, the spectral density of the peak is distributed over a finite frequency range $\Delta f_{\text {ac }}$, which is less than $0.4 \mathrm{~Hz}$. The peak intensity $I=\sum_{i} I_{i}$ is therefore determined by addition of all the spectral contributions $I_{i}$ spread from both sides of $f_{0}$ over $\Delta f_{\mathrm{ac}}$ and from which the thermal contribution $S_{z}(f)$ [see Eq. (2)] is subtracted. This provides an estimation of the force sensitivity (in newtons per square-root hertz), given by $\sqrt{8 \pi^{2} \gamma^{2} I\left(f_{0}^{2}+f_{T}^{2}\right)}$ according to Eq. (4).

Taking the minimal measurable peak spectral density $I_{\min }$ as one standard deviation $\sigma_{z}(f)$ of the PSD, we expect the sensitivity of the optical force microscope to be thermally limited at $\sqrt{8 \pi^{2} \gamma^{2} \sigma_{z}(f)\left(f_{0}^{2}+f_{T}^{2}\right)}$, which equals $2 \sqrt{2 k_{\mathrm{B}} T \gamma}$, using the property that for a continuous response driven by Gaussian white noise, the standard deviation of the power spectral density equals its value $\left[\sigma_{z}(f)=S_{z}(f)\right]$ [28]. With Gaussian white noise, therefore, the thermal force sensitivity depends only on the fluid properties and the radius of the NP via the Stokes drag, as for an AFM, where reducing dissipation sources is a key target to increase resolution [37]. In this respect, the possibility for trapping a Au NP of radius $R=75 \mathrm{~nm}$ is a good compromise between the $\sqrt{R}$ dependence of the Stokes-drag contribution, which must be reduced as much as possible to increase the thermally limited force sensitivity, and the $R^{3}$ dependence of the absorption cross section, which determines the strength of the radiation pressure.

In practice, starting with large $F_{\text {ac }}$ values, one first measures over the optimal bandwidth $\Delta f_{\text {opt }}$ the ac force signal at $f_{0}$ through a high peak spectral intensity $I \gg \sigma_{z}(f)$. Figure 3(a) gathers such force measurements obtained with a single trapped Au NP for a relatively large optical modulation ratio. We stress that all $f_{0}$ peak spectral intensities $S_{z}^{d}(f)$ are measured from a PSD (or with a lockin amplifier, as discussed in Supplemental Material Sec. A) acquired with a bandwidth of $\Delta f_{\text {opt }}=0.1 \mathrm{~Hz}$, hence at noise levels similar to the noise level of the PSD shown in Fig. 2.

Reducing the optical modulation ratio, one faces a relative increase of unavoidable noise (thermal, external, vibrations, etc.) with respect to the force signal. This noise contribution is analyzed through Allan-deviation analysis. After $10 \mathrm{~s}$, this deviation (seen in the inset in Fig. 2) reaches, at worst, twice the thermal contribution. This sets the optimal experimental sensitivity to $2 \times\left(2 \sqrt{2 k_{B} T \gamma}\right)=$ $9.2 \mathrm{fN} / \sqrt{\mathrm{Hz}}$ for our experimental conditions (single $\mathrm{Au}$ $\mathrm{NP}$, radius $75 \mathrm{~nm}$, trapped in water at room temperature) [38]. This sensitivity is valid only for experiments with timescale shorter than $\tau_{\mathrm{opt}}$.

Working at the optimal bandwidth $\Delta f_{\text {opt }}=0.1 \mathrm{~Hz}$, we expect in these conditions a resolution of $2.9 \mathrm{fN}$, which corresponds to the minimal force that can be measured by our microscope. All measurements of external forces below $2 F_{\text {th }}$ are discarded because the corresponding

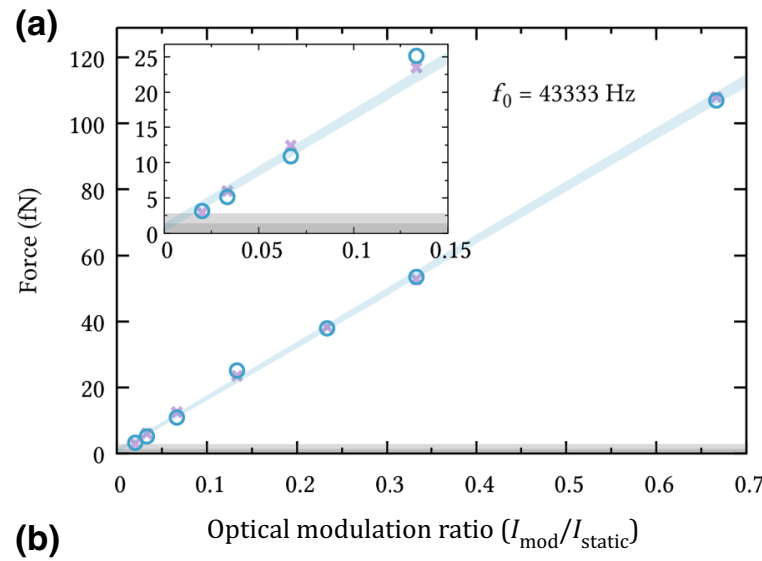

FIG. 3. (a) Optical forces measured at the modulation frequency $f_{0}=43333 \mathrm{~Hz}$ as a function of the ratio between the modulation of the pushing-beam amplitude $I_{\text {mod }}$ set by the function generator and the static pressure contribution $I_{\text {static }}$. The force is measured either by the intensity peak on the PSD plot (pink crosses) or with a lock-in amplifier (blue circles; see Supplementary Material Sec. A for details). The force, as expected, varies linearly (blue shade) with the pushing-laser modulation intensity. External forces down to approximately $3 \mathrm{fN}$ are measured with both the lock-in amplifier and the PSD with the relevant spectral bins populated by the external force and distributed over $\Delta f_{\mathrm{ac}}$. The force resolution is independent of the static dc radiationpressure component $F_{\mathrm{dc}}$. The smallest force measured $F_{\mathrm{ac}}^{\min }$ is almost 50 times smaller than $F_{\mathrm{dc}}$ determined to be approximately $160 \mathrm{fN}$ from the slope of the force versus modulation ratio. (b) Evolution of the smallest measured force obtained by both PSD and lock-in measurements at different drive frequencies $f_{0}$ (going from approximately $1 \mathrm{kHz}$ to approximately $100 \mathrm{kHz}$ ). The smallest measured force stays constant at ca. $3 \mathrm{fN}$ throughout the whole drive-frequency range ( 2 full orders of magnitude) with both methods. Dark and light shaded areas represent one and two thermal forces of $F_{\text {th }}$ and $2 F_{\text {th }}$, respectively, at the chosen bandwidth $\left(\Delta f_{\text {opt }}=0.1 \mathrm{~Hz}\right)$.

force signal cannot be discriminated from the noise [39]. Remarkably, as seen in the inset in Fig. 3(a), our system enables us to measure, directly from the $f_{0}$ modulation peak of the PSD, radiation pressures down to $3 \mathrm{fN}$ (i.e., at the level of the expected resolution). In agreement with these values, a Mie computation with field intensities estimated at the experimental limit yields a force of $4 \mathrm{fN}$ exerted on the $150-\mathrm{nm}$ Au sphere, a value in good agreement with our measurements. 
The accuracy of these values also depends on the precise determination of the bead radius. We use manufacturer specifications and have a $8 \%$ dispersion in the bead size. This variation of size induces a change in the viscosity that systematically shifts the values of all forces, including the thermal force.

We also verify that the experimental resolution is independent of the modulation frequency $f_{0}$, as expected from a Lorentzian PSD. Figure 3(b) gathers the smallest external forces measured from PSDs and with a lock-in amplifier for modulation frequencies taken below, at, and above the roll-off frequency $f_{T}$ of the optical trap. Remarkably, the values stay around $3 \mathrm{fN}$ (i.e., approximately 2 times larger than the thermal limit) regardless of the modulation frequency (i.e., in both the confined regime $f_{0}<f_{T}$ and the freely diffusing regime $f_{0}>f_{T}$ of the trap).

\section{POSITION NOISE}

Measuring $S_{z}^{d}(f)$ by selecting spectral bins of the PSD over a sufficiently narrow spectral bandwidth $\Delta f_{\text {ac }} \ll f_{0}$, or equivalently using a lock-in amplifier, as described in Supplemental Material Sec. A, corresponds to a band-pass filter centered on the modulation frequency $f_{0}$. In such conditions, the position noise is given by $\delta z_{\min } \sim \sqrt{\Delta f S_{z}\left(f_{0}\right)}$

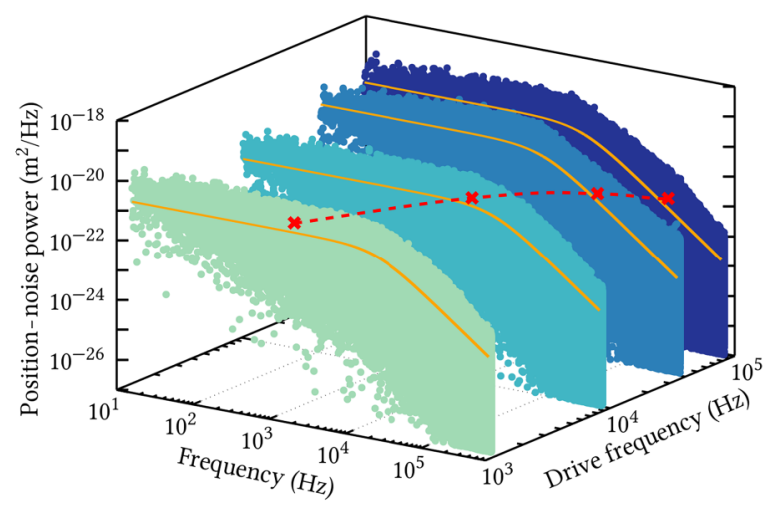

FIG. 4. Isometric representation of the PSD as a function of the external-drive frequency $f_{0}$ (going from approximately $1 \mathrm{kHz}$ to approximately $100 \mathrm{kHz}$ ). The position resolution measured by the lock-in amplifier (and converted back to noise powers) are superimposed on the graph as crosses as a function of $f_{0}$. The smallest measured displacement amplitude is less than $1 \AA$ and diminishes even further as the drive frequency departs from the Lorentzian plateau and reaches the free Brownian regime $\left(f^{-2}\right.$ at high frequencies in the PSD plot). The fit line (in orange) in each PSD plot represents the thermal contribution to the displacement. All measurements are acquired over thermally limited acquisition time $\tau_{\mathrm{opt}}=10 \mathrm{~s}$. Experiments are performed with different nanospheres and at different distances from the mirror. This results in different trap stiffnesses for the different series acquired. The roll-off frequencies are $17 \mathrm{kHz}$ for $f_{0}=1331 \mathrm{~Hz}$, $18.5 \mathrm{kHz}$ for $f_{0}=10331 \mathrm{~Hz}, 8 \mathrm{kHz}$ for $f_{0}=43333 \mathrm{~Hz}$, and 7.2 $\mathrm{kHz}$ for $f_{0}=97579 \mathrm{~Hz}$.
[40]. In contrast with the thermal limit for force measurements, position noises therefore depend on the modulation frequency.

Displacements associated with the smallest measured external forces are displayed in Fig. 4. As clearly seen, they lie within the PSD noise levels, separated by a factor of only approximately 2 from the Lorentzian fits. The $f_{0}$ dependence yields $\delta z_{\min }$, which rapidly decreases with $f^{2}$ as soon as the free Brownian regime is dominant for $f_{0}>$ $f_{T}$. Furthermore, the large $f_{T}$ values provide subangstrom levels of position resolution for all drive frequencies $f_{0}$ and displacements of $10^{-11} \mathrm{~m}$ reached at $f_{0} \sim 100 \mathrm{kHz}$.

\section{CONCLUSION}

The careful assessment of the conditions for stability of our experiment through Allan-deviation analysis validates our setup as a high-resolution optical force microscope. Over a thermally limited bandwidth of $0.1 \mathrm{~Hz}$, we are able to consistently measure radiation pressure down to approximately $3 \mathrm{fN}$. This result should also be appreciated in relation to a dynamical range $F_{\mathrm{ac}} / F_{\mathrm{dc}}$ of approximately 2 orders of magnitudes. This range, together with the capacity to reach femtonewton force-resolution levels in water at room temperature with relatively short acquisition times in the absence of any induced heating, is particularly important when one is aiming at studying new types of optical force fields [41], in particular in the context of evanescent and surface-plasmon optics [42,43], optical spin-orbit interactions [44,45], and chiral optical forces [46-49].

The concomitant subangstrom displacement resolution offered by our setup also opens new possibilities in the context of short-distance forces, such as Casimir-like interactions [1] or optical binding effects [50], where adjustable roll-off frequencies allow tuning of the diffusion volume of the trapped NP, and thereby giving a capacity of localization on nanometer scales. This capacity could be important for resolving nonlinear force signals, such as found at the level of self-organized supramolecular assemblies in mechanochemistry [51]. In this context, the reliability and resolution provided by our force microscope could help in exploring connections between optical force and chemical signals.

\section{ACKNOWLEDGMENTS}

This work was supported in part by Agence Nationale de la Recherche (ANR), France, ANR Equipex Union (Grant No. ANR-10-EQPX-52-01), the Labex NIE projects (Grant No. ANR-11-LABX-0058-NIE), and USIAS within the Investissements d'Avenir program (Grant No. ANR10-IDEX-0002-02). Y.R.-C. is a member of the International Doctoral Program of the Initiative d'Excellence of the University of Strasbourg, whose support is acknowledged. 


\section{APPENDIX A: LOCK-IN DETECTION METHOD}

When one is measuring forces, in particular via the determination of the position noise $S_{z}^{d}\left(f_{0}\right)$ [see Eq. (4)], it is convenient to resort to a time-domain measurement such as a lock-in detection method [52]. Experimentally, $S_{z}^{d}\left(f_{0}\right)$ is measured from the intensity signal passing through a lownoise preamplifier, with the signal then being sent to both an acquisition card and a lock-in amplifier, as described in Fig. 5.

The lock-in amplifier immediately provides the spectral power signal $S_{z}^{d}\left(f_{0}\right)$. However, its estimation from the complete bead-displacement PSD can be computed off-line directly from the recorded intensity-time trace saved on a computer. The lock-in method is a viable alternative only when its output signal is calibrated.

This calibration is not straightforward since the lockin amplifier mixes, in the time domain, the contributions of both the thermal fluctuations and the external ac drive, which cannot therefore be isolated from one another. But the lock-in calibration can be performed through the results obtained with the self-calibrated PSD approach at high modulation amplitudes - modulation ratios greater than 0.1 in Fig. 3(a) - where all noise contributions to the signal can be ignored. Both methods (PSD and lock-in amplifier) provide values proportional to $S_{z}^{d}\left(f_{0}\right)$. In this regime of strong drive, the linearity of the lock-in output signal is calibrated to the linearity of the power spectral intensity peak at $f_{0}$ measured from the PSD and converted, as $F_{\mathrm{ac}}$, to newtons, as discussed above. The experimental output signals are displayed in Fig. 3 and are superimposed on those obtained by the PSD method. The calibrated lockin may relieve the constraint of identifying spectral bins populated by the external excitation, but in our case both approaches are strictly equivalent. Spectral bins are well

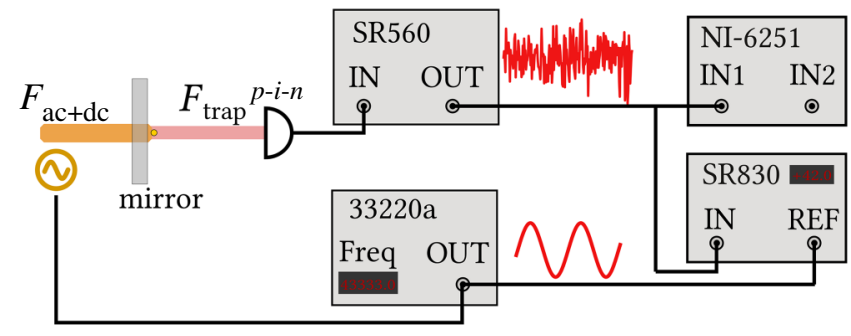

FIG. 5. The experimental setup used to measure $S_{z}^{d}\left(f_{0}\right)$, Eq. (4). The voltage recorded by the photodiode $(p-i-n)$ is sent to a lownoise preamplifier (SR560, Stanford Research). The signal is filtered to remove its dc component with a high-pass filter with a cutoff frequency of $0.03 \mathrm{~Hz}$. It is then sent to both a 16-bit acquisition card (NI-6251, National Instruments) and a lockin amplifier (SR830, Stanford Research). A function generator (33220A, Agilent Technologies) produces a sinusoidal output of amplitude and frequency controlled by a computer. The sine function is sent simultaneously to the pushing-laser controller and the lock-in amplifier as the reference. identified, even for low external drives. This equivalence is clearly seen in Fig. 3, where PSD values match well with lock-in values.

When the modulation amplitude of the pushing laser is reduced, we still measure a proportional force amplitude up to a few femtonewtons, as observed in the inset in Fig. 3 (a). In these conditions, it is possible to perform measurements down to the $2 F_{\text {th }}$ limit, below which noise sources dominate over the external force. Figure 3(a) shows that the smallest external force we measure using the lock-in amplifier is approximately $3 \mathrm{fN}$ (i.e., similar than the level reached directly with the PSD).

\section{APPENDIX B: FORCE MEASUREMENTS AT DIFFERENT MODULATION FREQUENCIES}

The external force is measured at different modulation (driving) frequencies $f_{0}$ corresponding to confined $\left(f_{0}<f_{T}\right)$ or freely diffusing $\left(f_{0}>f_{T}\right)$ Brownian motion within the trap. For an overdamped Brownian particle solely driven by thermal fluctuations, the measured external force, and in our case the minimal measurable external force, is expected to remain constant regardless of the modulation frequency. This is verified experimentally with modulation frequencies $f_{0}$ of 1331, 10331,43333 , and $97579 \mathrm{~Hz}$, spanning 2 orders of magnitude and crossing through the trap roll-off frequency. The results are shown in Fig. 6.

For these experiments, an external driving force is applied on single 150-nm Au NPs trapped a few microns from the mirror. The amplitude of the drive is modulated with respect to the mean intensity of the optical pushing beam $(\lambda=639 \mathrm{~nm})$. The crosses in Fig. 6 indicate external-force values extracted from the PSDs and the circles represent forces measured by the lock-in amplifier after its response is linearly calibrated for strong external drives (see Appendix A). We stress that each value is recorded over the same bandwidth $\Delta f=0.1 \mathrm{~Hz}$. The linearity of measured forces with modulation amplitudes is apparent and is quantified by an uncertainty interval (a deviation from linearity of $\pm 1 \sigma$ ) on the whole measurement series in blue shades. Gray areas correspond to thermal-force noise floors at one and two standard deviations for dark and light regions, respectively.

All recorded series display external forces that are measured above the stringent resolution criterion of $2 F_{\text {th }}$ that we select (i.e., $2.9 \mathrm{fN}$ ). Remarkably, while the bandwidth is chosen to account for the worst-case stability scenario for a single NP, the good linearity of the overall series (taking up to a few minutes) suggests that longer acquisition times do not deviate much, for good series, from the Allan variance at $10 \mathrm{~s}$. This could allow even shorter bandwidth when one is considering single measurements, although such a possibility must be proved through a longtime Allan stability analysis of the system. This, however, 

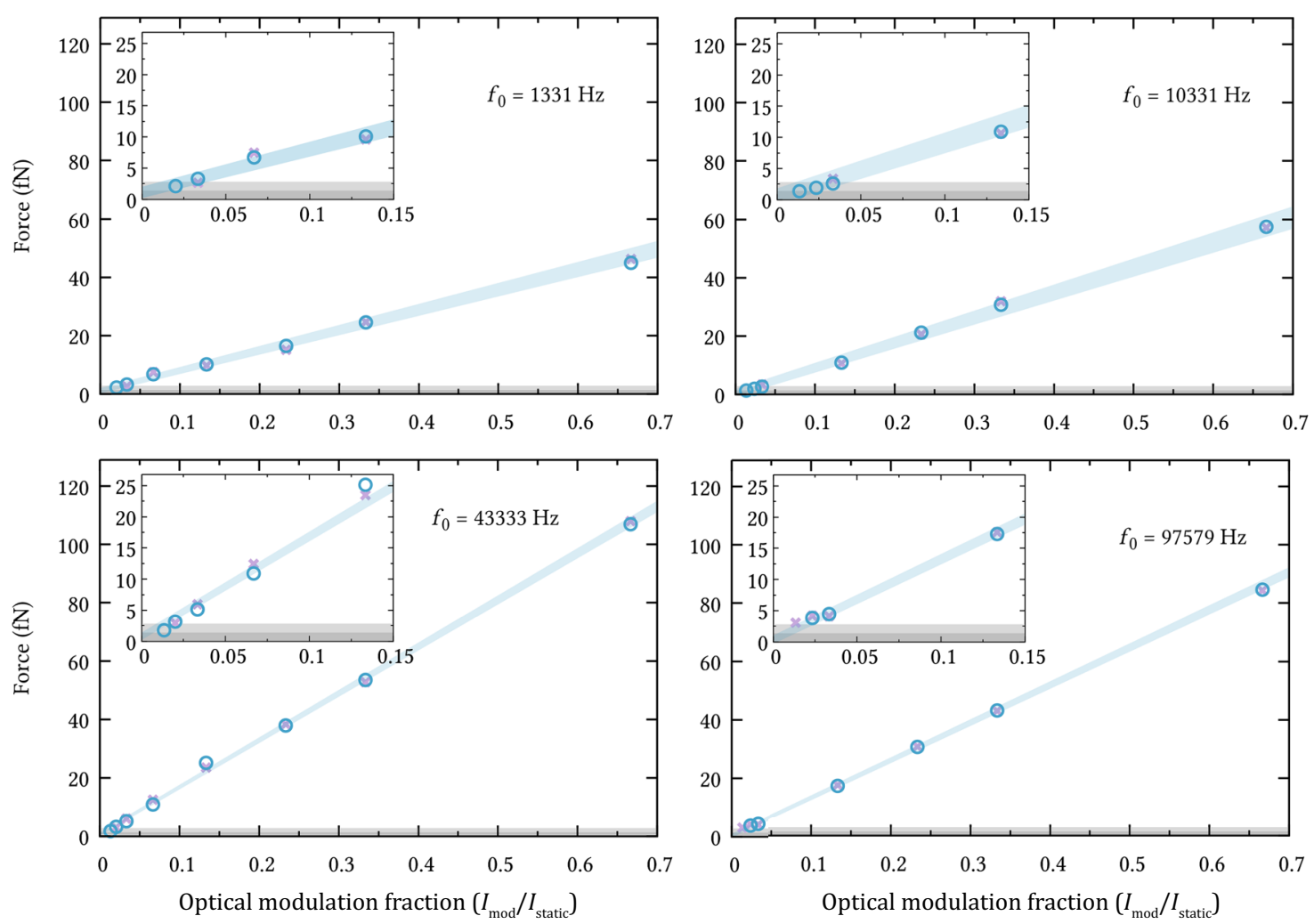

FIG. 6. Measured external forces driven at $f_{0}$ exerted on optically trapped single Au NPs of $150 \mathrm{~nm}$ at frequencies of 1331,10331 , 43333 , and $97579 \mathrm{~Hz}$. The amplitude of the drive is modulated with respect to the mean intensity of the optical beam $(\lambda=639 \mathrm{~nm})$. The crosses are measured external forces from the PSDs and the circles represent forces measured by the lock-in amplifier after its response is linearly calibrated from strong external drives. Each value has a bandwidth $\Delta f=0.1 \mathrm{~Hz}$. The linearity of measured forces with modulation amplitude is quantified through an uncertainty interval (a deviation from linearity of $\pm 1 \sigma$ ) on the whole measurement series. Dark and light shaded areas represent one and two thermal forces of $F_{\text {th }}$ and $2 F_{\text {th }}$, respectively, at the chosen bandwidth $(0.1$ $\mathrm{Hz})$.

is difficult to implement with our colloidal suspensions and our acquisition card.

\section{APPENDIX C: HEATING EFFECTS}

Heating effects can be induced by the temperature elevation at the surface of the metallic sphere under laser irradiation. The rise in temperature can be estimated by our considering the radius $R$ and the absorption cross section $\sigma_{\text {abs }}$ of the sphere, as well as the water thermal conductivity $k_{s}$ and the irradiance $I$ [22]:

$$
\Delta T=\frac{\sigma_{\mathrm{abs}} I}{4 \pi k_{S} R} .
$$

Such effects have been measured experimentally by looking, for instance, at trap stiffness variations [53], shifts of the localized plasmon resonances [54], or thermal damaging of supporting membranes [55]. These experiments provide thermal elevation of approximately $500 \mathrm{~K} / \mathrm{W}$ for $\mathrm{Au}$ spheres of $100 \mathrm{~nm}$ under Gaussian illumination at a wavelength of $1064 \mathrm{~nm}$, in relatively good agreement with Eq. (C1).

Adjustment of this value for our 150-nm spheres illuminated at $785 \mathrm{~nm}$ provides temperature elevations of more than $1500 \mathrm{~K} / \mathrm{W}$, which corresponds to an increase of $40 \mathrm{~K}$ with our approximately-25-mW laser. Because the viscosity of water $\eta=\eta(T)$ is strongly dependent on temperature variations, this increase is expected to lead to a factorof-2 change in the viscosity $-\eta(T=300 \mathrm{~K})=0.85$ and $\eta(T=340 \mathrm{~K})=0.42$ - that necessarily would alter our external-force estimation by the same factor.

However, such a change is not observed in our experiments. We carefully check this by varying, through a rotating optical density, the trapping-laser power for single $\mathrm{Au}$ NPs $(150 \mathrm{~nm})$ trapped at different distances from the mirror. As clearly seen in Fig. 7, the roll-off frequencies for all trapping conditions follow a linear behavior. This behavior is expected in the absence of heating effects, considering that the trapping roll-off frequency $f_{T}$ is directly proportional to the trapping-laser intensity. From the trap 


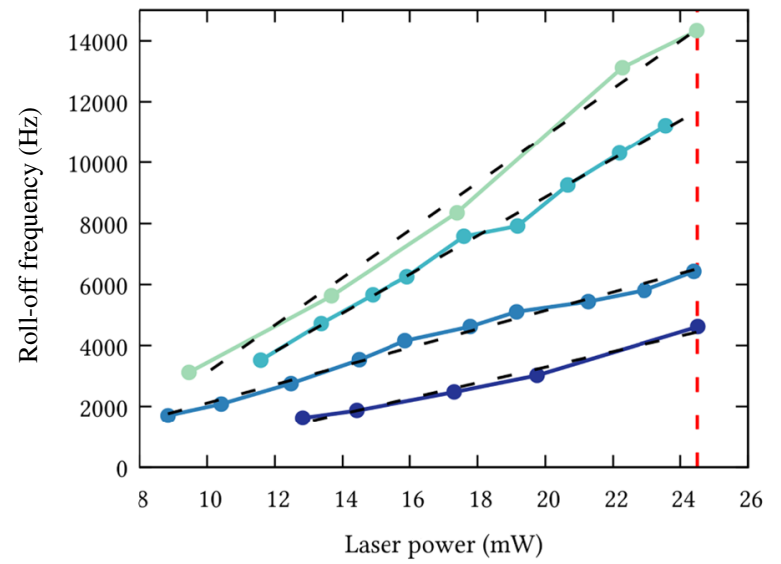

FIG. 7. Evolution of the trap stiffness with incident trappinglaser powers for four different $\mathrm{Au}$ spheres of $150 \mathrm{~nm}$ and at different stable trapping positions. Regardless of the mirror-waist distances, the trap stiffnesses, while different, increase linearly with increasing laser power. The behavior is expected when the drag term $\gamma$ remains constant. This implies that the viscosity of the fluid, and therefore its temperature, does not evolve sufficiently to be detected within the trapping-laser power range explored here. This verifies that the possible heating of the trapped NPs remains small enough as to not induce any significant variations of the surrounding viscosity. The second curve starting from the top is offset by $-1000 \mathrm{~Hz}$ to ease readability. The dashed red line $(24.5 \mathrm{~mW})$ corresponds to the trapping-laser intensity that is used in our experiments.

stiffness $\kappa_{T}=12 \pi^{2} \eta(T) R f_{T}$, this linear dependence shows that the viscosity $\eta(T)$ of water inside the trap must remain constant throughout the variation of intensity. We can therefore conclude that there is no significant heating effect on the dynamics of the trapped object in our experiments.

Trapping of a spherical Au NP in a standing-wave optical trap is characterized by complex patterns of stability regions [19]. In our experiments, the absence of heating leads to our inferring that the NP is trapped outside fieldintensity maxima of the standing wave. Our system thus appears analogous to a cage with optical walls along the optical axis, preventing the NP from crossing antinodes of the interference pattern, while being confined transversely by the residual gradient contributions of the trapping beam.

Finally, no changes are observed in the trap stiffness with the external $\mathrm{dc}$ force present. The external pushing field is a few orders of magnitude weaker than the trapping beam and does not induce changes in the fluid properties despite its higher absorption cross section at the pushing-laser wavelength.

\section{APPENDIX D: SINUSOIDAL FORCING}

In our experiments, external forces applied on the optically trapped metallic nanospheres are generated by sinusoidal forcing. This means that, relative to a mean position

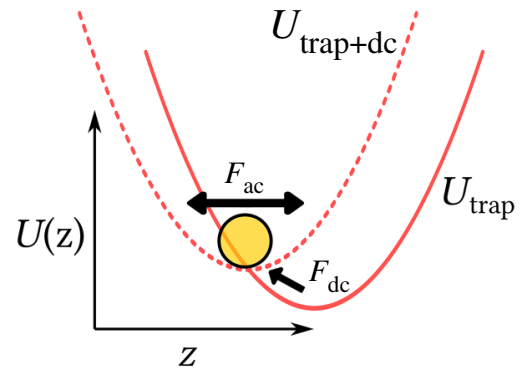

FIG. 8. Simplified energy landscape of the trapped Au nanosphere under an oscillating external force. This contribution consists of a sinusoidal modulation of the pushing-laser intensity around a constant value. As a consequence, the resulting force applied on the sphere consists of two terms: $F_{\mathrm{dc}}$ and $F_{\mathrm{ac}}$. The constant contribution $\left(F_{\mathrm{dc}}\right)$ shifts the equilibrium position of the trapped object (solid line) into a new effective harmonic potential (dashed line) and equilibrium position. The oscillating term $\left(F_{\mathrm{ac}}\right)$ at $f_{0}$ will sinusoidally push and pull the confined metallic sphere with respect to this new equilibrium position.

set by the static (dc) contribution of the forcing, the sinusoidal modulation effectively pushes and pulls the particle every half period. This is achieved experimentally by our modulating the intensity of the pushing laser sinusoidally around a mean value. The resulting beam intensity thus includes static (dc) and dynamic (ac) components. The static contribution $F_{\mathrm{dc}}$ will push the sphere and displace it with respect to the initial trapping potential. The Gaussian dynamics of the sphere displacements is preserved but the equilibrium position is shifted along the optical axis. This amounts to our defining a new (shifted) effective attractive potential for the bead motion, drawn as a dashed line in Fig. 8. The dynamic contribution $F_{\text {ac }}$ to the force moves the particle back and forth sinusoidally in this new effective potential.

It is worth mentioning that this description matches perfectly the dynamics observed experimentally. It is clear from Fig. 2 that even for high external-modulation strengths, no harmonics of the drive frequency $f_{0}$ are observed in the spectral signatures of the PSD. This contrasts with previous work where the periodic excitation was created by a chopper to modulate the signal on or off $[3,4]$. In this case, odd harmonics of $f_{0}$ are necessarily present in the PSD, and the measured $S_{z}^{d}(f)$ amplitude has to be corrected for them to account for the square nature of the driving signal.

[1] D. S. Ether, Jr., L. B. Pires, S. Umrath, D. Martinez, Y. Ayala, B. Pontes, G. R. de S. Araújo, S. Frases, G.-L. Ingold, F. S. S. Rosa, N. B. Viana, H. M. Nussenzveig, and P. A. Maia Neto, Probing the Casimir force with optical tweezers, Europhys. Lett. 112, 44001 (2015). 
[2] L. Helden, R. Eichhorn, and C. Bechinger, Direct measurement of thermophoretic forces, Soft Matter 11, 2379 (2015).

[3] C. Zensen, N. Villadsen, F. Winterer, S. R. Keiding, and T. Lohmüller, Pushing nanoparticles with light - a femtonewton resolved measurement of optical scattering forces, APL Photonics 1, 026102 (2016).

[4] L. Liu, S. Kheifets, V. Ginis, and F. Capasso, Subfemtonewton Force Spectroscopy at the Thermal Limit in Liquids, Phys. Rev. Lett. 116, 228001 (2016).

[5] K. C. Neuman and A. Nagy, Single-molecule force spectroscopy: Optical tweezers, magnetic tweezers and atomic force microscopy, Nat. Meth. 5, 491 (2008).

[6] S. Ciliberto, S. Joubaud, and A. Petrosyan, Fluctuations in out-of-equilibrium systems: From theory to experiment, J. Stat. Mech. 2010, P12003 (2010).

[7] O. M. Maragò, P. H. Jones, P. G. Gucciardi, G. Volpe, and A. C. Ferrari, Optical trapping and manipulation of nanostructures, Nat. Nano 8, 807 (2013).

[8] P. Wu, R. Huang, C. Tischer, A. Jonas, and E.-L. Florin, Direct Measurement of the Nonconservative Force Field Generated by Optical Tweezers, Phys. Rev. Lett. 103, 108101 (2009).

[9] L. Perez Garcia, J. Donlucas Perez, G. Volpe, A. V. Arzola, and G. Volpe, High-performance reconstruction of microscopic force fields from Brownian trajectories, arXiv:1808.05468 (2018).

[10] V. Svak, O. Brzobohatỳ, M. Siler, P. Jákl, J. Kanka, P. Zemánek, and S. H. Simpson, Transverse spin forces and non-equilibrium particle dynamics in a circularly polarized vacuum optical trap, Nat. Commun. 9, 5453 (2018).

[11] L. Tong, V. D. Miljkovic, and M. Käll, Alignment, rotation, and spinning of single plasmonic nanoparticles and nanowires using polarization dependent optical forces, Nano Lett. 10, 268 (2009).

[12] D. Rings, R. Schachoff, M. Selmke, F. Cichos, and K. Kroy, Hot Brownian Motion, Phys. Rev. Lett. 105, 090604 (2010).

[13] P. V. Ruijgrok, N. R. Verhart, P. Zijlstra, A. L. Tchebotareva, and M. Orrit, Brownian Fluctuations and Heating of an Optically Aligned Gold Nanorod, Phys. Rev. Lett. 107, 037401 (2011).

[14] A. Cuche, B. Stein, A. Canaguier-Durand, E. Devaux, C. Genet, and T. W. Ebbesen, Brownian motion in a designer force field: Dynamical effects of negative refraction on nanoparticles, Nano Lett. 12, 4329 (2012).

[15] P. V. Ruijgrok, P. Zijlstra, A. L. Tchebotareva, and M. Orrit, Damping of acoustic vibrations of single gold nanoparticles optically trapped in water, Nano Lett. 12, 1063 (2012).

[16] A. Ohlinger, A. Deak, A. A. Lutich, and J. Feldmann, Optically Trapped Gold Nanoparticle Enables Listening at the Microscale, Phys. Rev. Lett. 108, 018101 (2012).

[17] K. Svoboda and S. M. Block, Optical trapping of metallic Rayleigh particles, Opt. Lett. 19, 930 (1994).

[18] P. M. Hansen, V. K. Bhatia, N. Harrit, and L. B. Oddershede, Expanding the optical trapping range of gold nanoparticles, Nano Lett. 5, 1937 (2005).

[19] M. Šiler, L. Chvátal, and P. Zemánek, Metallic nanoparticles in a standing wave: Optical force and heating, J. Quant. Spectrosc. Radiat. Transf. 126, 84 (2013).
[20] J. R. Moffitt, Y. R. Chemla, S. B. Smith, and C. Bustamante, Recent advances in optical tweezers, Annu. Rev. Biochem. 77, 205 (2008).

[21] S. Lal, S. Link, and N. J. Halas, Nano-optics from sensing to waveguiding, Nat. Photon. 1, 641 (2007).

[22] G. Baffou and R. Quidant, Thermo-plasmonics: Using metallic nanostructures as nano-sources of heat, Laser Photon. Rev. 7, 171 (2012).

[23] P. Samori, Scanning Probe Microscopies Beyond Imaging: Manipulation of Molecules and Nanostructures (WileyVCH, Weinheim, 2006).

[24] G. Jourdan, A. Lambrecht, F. Comin, and J. Chevrier, Quantitative non-contact dynamic Casimir force measurements, Europhys. Lett. 85, 31001 (2009).

[25] D. Guan, Z. H. Hang, Z. Marcet, H. Liu, I. Kravchenko, C. T. Chan, H. B. Chan, and P. Tong, Direct measurement of optical force induced by near-field plasmonic cavity using dynamic mode AFM, Sci. Rep. 5, 16216 (2015).

[26] S. F. Tolic-Norrelykke, E. Schäffer, J. Howard, F. S. Pavone, F. Juelicher, and H. Flyvbjerg, Calibration of optical tweezers with positional detection in the back focal plane, Rev. Sci. Inst. 77, 103101 (2006).

[27] E. Hebestreit, M. Frimmer, R. Reimann, C. Dellago, F. Ricci, and L. Novotny, Calibration and energy measurement of optically levitated nanoparticle sensors, Rev. Sci. Inst. 89, 033111 (2018).

[28] K. Berg-Sørensen and H. Flyvbjerg, Power spectrum analysis for optical tweezers, Rev. Sci. Inst. 75, 594 (2004).

[29] F. Czerwinski, A. C. Richardson, and L. B. Oddershede, Quantifying noise in optical tweezers by Allan variance, Opt. Express 17, 13255 (2009).

[30] D. T. Edwards, J. K. Faulk, A. W. Sanders, M. S. Bull, R. Walder, M.-A. LeBlanc, M. C. Sousa, and T. T. Perkins, Optimizing 1- $\mu \mathrm{m}$-resolution single-molecule force spectroscopy on a commercial atomic force microscope, Nano Lett. 15, 7091 (2015).

[31] G. I. Harris, D. L. McAuslan, T. M. Stace, A. C. Doherty, and W. P. Bowen, Minimum Requirements for Feedback Enhanced Force Sensing, Phys. Rev. Lett. 111, 103603 (2013).

[32] G. Schnoering, Ph.D. thesis, University of Strasbourg, 2016.

[33] P. Zemánek, A. Jonáš, L. Šrámek, and M. Liška, Optical trapping of nanoparticles and microparticles by a Gaussian standing wave, Opt. Lett. 24, 1448 (1999).

[34] O. Brzobohatỳ, M. Šiler, J. Trojek, L. Chvátal, V. Karásek, A. Paták, Z. Pokorná, F. Mika, and P. Zemánek, Threedimensional optical trapping of a plasmonic nanoparticle using low numerical aperture optical tweezers, Sci. Rep. 5, 8106 (2015).

[35] P. Welch, The use of fast Fourier transform for the estimation of power spectra: A method based on time averaging over short, modified periodograms, IEEE Trans. Audio Electroacoust. 15, 70 (1967).

[36] D. W. Allan, Statistics of atomic frequency standards, Proc. IEEE 54, 221 (1966).

[37] A. N. Cleland and M. L. Roukes, Noise processes in nanomechanical resonators, J. Appl. Phys. 92, 2758 (2002).

[38] Constrained by the Allan-variance analysis, we do acquire our results under the same conditions as in Ref. [4]. We limit ourselves to a single series of measurements acquired 
over $10 \mathrm{~s}$ only. This warrants our smallest force measurements being as close as possible to the thermal limit.

[39] Averaging the PSD necessarily reduces the frequency bandwidth because each signal series involved in the averaging is measured on a shorter timescale. As a consequence of this reduction in bandwidth, the width of the peak associated with the external modulated force is increased and its height is reduced. This implies that the improvement associated with the reduction of the PSD variance is lost when the peak is measured at a smaller bandwidth. For a system without additional external noise sources, both approaches (full signal vs extracted series) should give the same measurement on external displacement. However, in our configuration, averaging the spectral density can lead to increasing errors in the measured force. As it is seen from the Allan deviation, one position measurement performed over $1 \mathrm{~s}$ is already not solely thermally driven. Averaging our signal acquired over $10 \mathrm{~s}$ as $10 \times 1 \mathrm{~s}$ will necessarily add the nonthermal noise observed at $1 \mathrm{~s}$. Because the ratios between the signal measured and the thermal floor due to other noise contributions are comparable between 1 and 10 $\mathrm{s}$, the errors would simply be 10 times larger on the averaged position dynamics than for a single measurement of 10 s. In our case therefore, averaging the PSD cannot provide any improvement in the force measurement.

[40] S. Reynaud, Introduction à la réduction du bruit quantique, Ann. Phys. Fr. 15, 63 (1990).

[41] D. B. Ruffner and D. G. Grier, Optical Forces and Torques in Nonuniform Beams of Light, Phys. Rev. Lett. 108, 173602 (2012).

[42] F. J. Rodríguez-Fortuño, N. Engheta, A. Martínez, and A. V. Zayats, Lateral forces on circularly polarizable particles near a surface, Nat. Commun. 6, 8799 (2015).

[43] L. Liu, S. Kheifets, V. Ginis, A. Di Donato, and F. Capasso, Elliptical orbits of microspheres in an evanescent field, Proc. Natl. Acad. Sci. U. S. A. 114, 11087 (2017).

[44] S. Sukhov, V. Kajorndejnukul, R. R. Naraghi, and A. Dogariu, Dynamic consequences of optical spin-orbit interaction, Nat. Photon. 9, 809 (2015).
[45] L. Liu, A. Di Donato, V. Ginis, S. Kheifets, A. Amirzhan, and F. Capasso, Three-Dimensional Measurement of the Helicity-Dependent Forces on a Mie Particle, Phys. Rev. Lett. 120, 223901 (2018).

[46] A. Canaguier-Durand, J. A. Hutchison, C. Genet, and T. W. Ebbesen, Mechanical separation of chiral dipoles by chiral light, New J. Phys. 15, 123037 (2013).

[47] R. P. Cameron, S. M. Barnett, and A. M. Yao, Discriminatory optical force for chiral molecules, New J. Phys. 16, 013020 (2014).

[48] K. Ding, J. Ng, L. Zhou, and C. T. Chan, Realization of optical pulling forces using chirality, Phys. Rev. A 89, 063825 (2014).

[49] G. Schnoering, L. V. Poulikakos, Y. Rosales-Cabara, A. Canaguier-Durand, D. J. Norris, and C. Genet, Three-Dimensional Enantiomeric Recognition of Optically Trapped Single Chiral Nanoparticles, Phys. Rev. Lett. 121, 023902 (2018).

[50] K. Dholakia and P. Zemánek, Colloquium: Gripped by light: Optical binding, Rev. Mod. Phys. 82, 1767 (2010).

[51] C. R. Hickenboth, J. S. Moore, S. R. White, N. R. Sottos, J. Baudry, and S. R. Wilson, Biasing reaction pathways with mechanical force, Nature 446, 423 (2007).

[52] T. Kouh, U. Kemiktarak, O. Basarir, C. Lissandrello, and K. L. Ekinci, Measuring Gaussian noise using a lock-in amplifier, Am. J. Phys. 82, 778 (2014).

[53] Y. Seol, A. E. Carpenter, and T. T. Perkins, Gold nanoparticles: Enhanced optical trapping and sensitivity coupled with significant heating, Opt. Lett. 31, 2429 (2006).

[54] A. Andres-Arroyo, F. Wang, W. J. Toe, and P. Reece, Intrinsic heating in optically trapped Au nanoparticles measured by dark-field spectroscopy, Biomed. Opt. Express 6, 3646 (2015).

[55] P. M. Bendix, S. N. S. Reihani, and L. B. Oddershede, Direct measurements of heating by electromagnetically trapped gold nanoparticles on supported lipid bilayers, ACS Nano 4, 2256 (2010). 